\title{
John Henryism Active Coping, Acculturation, and Psychological Health in Korean Immigrants
}

Journal of Transcultural Nursing

I-II

(c) The Author(s) 2015

Reprints and permissions:

sagepub.com/journalsPermissions.nav

DOI: $10.1177 / 1043659615615402$

tcn.sagepub.com

(SAGE

\author{
Jeongok G. Logan, PhD, RN', \\ Debra J. Barksdale, PhD, RN, FNP-BC, ANP-BC, CNE, FAANP, FAAN², \\ Sherman A. James, PhD, FAHA ${ }^{3}$, and Lung-Chang Chien, DrPH ${ }^{4}$
}

\begin{abstract}
This study aimed to explore the levels of John Henryism $(\mathrm{JH})$ active coping and its association with acculturation status and psychological health (specifically perceived stress, acculturative stress, anxiety, and depression) in Korean immigrants to the United States. In 102 Korean immigrants, JH active coping was measured by the JH Scale; acculturation by the Bidimensional Acculturation Scale; perceived stress by the Perceived Stress Scale; acculturative stress by the Social, Attitudinal, Familial, and Environmental Scale; anxiety by the State Anxiety Subscale of the Spielberger State-Trait Anxiety Inventory; and depression by the Center for Epidemiological Studies Depression Scale. The levels of JH active coping in this sample of Korean immigrants appear to be lower than the levels reported in other racial groups. Independent of demographic factors, JH active coping was a significant predictor of higher acculturation status and better psychological health as indicated by lower levels of perceived stress, acculturative stress, anxiety, and depressive symptoms.
\end{abstract}

\section{Keywords}

John Henryism active coping, immigrants, acculturation, stress, anxiety, depression

\section{Introduction}

Immigration is a major life event that often generates high levels of psychological stress (Claassen, Ascoli, Berhe, \& Priebe, 2005; Fazel, Wheeler, \& Danesh, 2005). Among Korean immigrants specifically, studies have reported high levels of psychological stress associated with conflicts in values, beliefs, and behaviors between mainstream society and their own ethnic group (Chun, Knight, \& Youn, 2007; M. T. Kim, Han, Shin, Kim, \& Lee, 2005; Koh, 1998; Shin, 1993). In addition, psychological problems related to depression are commonly reported in Korean immigrants (Han, Kim, Lee, Pistulka, \& Kim, 2007; Jang, Kim, \& Chiriboga, 2005; Kuo, 1984; Lin et al., 1992). Although Korean immigrants have been reported to experience high levels of psychological stress and problems, their stress management or coping mechanisms remain unclear.

Coping is defined as thoughts and behaviors used to manage internal and external stressors (Lazarus \& Folkman, 1984). Coping characteristics of the individual can determine either adaptation or maladaptation to stressful life events (Folkman \& Moskowitz, 2004). John Henryism (JH) active coping is defined as a strong behavioral predisposition to confront daily psychosocial stressors in an active and effortful manner (James, Hartnett, \& Kalsbeek, 1983; James,
Strogatz, Wing, \& Ramsey, 1987). The construct is based on John Henry, a Black American folk hero known for his physical strength, endurance, and determination. He allegedly defeated a steam powered machine in a contest of "man against machine," but fell dead from mental and physical exhaustion after he won the battle (James et al., 1987). JH active coping is measured in an individual by the JH Active Coping Scale (James et al., 1983). The resulting JH active coping proposition predicts that individuals of lower socioeconomic status (SES; e.g., education, income, or occupation) who score high on the JH Active Coping Scale are more likely to have higher blood pressure than those who possess more socioeconomic resources (James, 1994; James et al., 1987). This has been supported by a number of studies

\footnotetext{
'University of Virginia, Charlottesville, VA, USA

${ }^{2}$ Virginia Commonwealth University, Richmond, VA, USA

${ }^{3}$ Emory University, Atlanta, GA, USA

${ }^{4}$ University of Texas School of Public Health at San Antonio Regional Campus, TX, USA
}

\section{Corresponding Author:}

Jeongok G. Logan, PhD, RN, Department of Acute \& Specialty Care, School of Nursing, University of Virginia, McLeod Building, Room 40II, 225 Jeanette Lancaster Way, Charlottesville, VA 22903, USA.

Email: j|3zj@virginia.edu 
examining the relationship between stress, active coping, and cardiovascular health in both non-U.S. Blacks and Black Americans (Duijkers, Drijver, Kromhout, \& James, 1988; A. F. Fernander, Duran, Saab, \& Schneiderman, 2004; Merritt, Bennett, Williams, Sollers, \& Thayer, 2004; Wigg, Wright, Breach, \& Wilson, 1996; Wiist \& Flack, 1992).

JH may also imply optimism and energy. Under certain circumstances and perhaps for certain ethnic groups, high JH active coping may be indicative of good physiological and psychological health. For example, JH active coping was reported to be protective of posttraumatic stress disorder in White women (Kramer, Johnson, \& Johnson, 2015) and to be associated with self-reported healthy behaviors in African American men and women (Lehto \& Stein, 2013; Orr, James, Garry, \& Newton, 2006) and self-reported physical health in African Americans (Bonham, Sellers, \& Neighbors, 2004). $\mathrm{JH}$ active coping is compatible with Western individualism, which places great emphasis on self-reliance and independence. Asian immigrants from a culture with a more collectivist orientation might score lower on $\mathrm{JH}$ active coping; the effects of this potential association on their acculturation process and psychological health are unknown. Studies of JH active coping among minority groups other than African Americans in the United States are very limited. To date, only one study has been conducted among Asian Americans, which focused on Chinese and Indian Americans with high SES (Haritatos, Mahalingam, \& James, 2007). Their study found that high JH scores were positively associated with higher self-reported physical health and lower perceived stress.

The purpose of this study was to determine the effect of $\mathrm{JH}$ active coping on acculturation and psychological health in Korean immigrants who reportedly have have high levels of psychological stress. The objectives of this study were (1) to measure levels of JH active coping among Korean immigrants and (2) to examine the associations of JH active coping with acculturation and psychological health (measured as perceived stress, acculturative stress, anxiety, and depression). In this sample of Korean immigrants, we hypothesized the following: (1) JH active coping scores will be lower than ones reported in previous studies, yet (2) JH active coping will be a significant predictor of acculturation and psychological health.

\section{Method}

\section{Subjects}

After receiving institutional review board approval from University of North Carolina at Chapel Hill, a convenience sample of 102 Korean immigrants, aged 21 to 60 years, was recruited from three counties in North Carolina via mass e-mail, flyers, and word of mouth. Since this study intended to assess stress associated with acculturation, only first-generation Korean immigrants were recruited. More specifically, Korean immigrants who were born in Korea and had lived in the United States for at least 1 year were invited to participate in the study. Only subjects who were able to speak and read Korean were recruited, and the consent form and all questionnaires were administered in Korean.

\section{Measures}

The variables measured include $\mathrm{JH}$ active coping, acculturation, psychological stress (operationalized by perceived stress, acculturative stress), stress-related emotions (operationalized by anxiety and depression), and demographic factors (age, gender, time in the United States, and SES defined by education and household income). Because psychological concepts are time and situation dependent, the temporal stability of the Korean version of the psychometric instruments were examined by test-retest reliability; the instruments were mailed to the subjects' homes 2 weeks after the date of the first data collection. Among 102 participants, 74 participants (response rate $=72.55 \%$ ) returned their scales within 2 weeks, and these responses were included for the analysis of test-retest reliability.

$\mathrm{JH}$ Active Coping. $\mathrm{JH}$ active coping was measured by the $\mathrm{JH}$ Active Coping Scale (James et al., 1987). This 12-item scale measures the behavioral predisposition to cope actively with difficult psychosocial stressors and barriers. According to James et al. (1987), the scale consists of three themes: a commitment to hard work, a determination to achieve one's goals, and efficacious mental and physical vigor. Each item is rated on a 5-point Likert-type scale ranging from 1 (completely false) to 5 (completely true); the total John Henryism scores range from 12 to 60 , with higher scores indicating a greater inclination to cope with perceived stressors actively, rather than passively. Haritatos et al. (2007) and A. F. Fernander et al. (2005) have reported Cronbach's alphas of .76, and .73, respectively. In the current study, the internal consistency of the JH Scale as measured by Cronbach's alpha was .79 , with test-retest reliability over 2 weeks of .61 .

Acculturation. Acculturation was measured using the American Orientation Subscale of the Bidimensional Acculturation Scale. The Bidimensional Acculturation Scale was developed by Jang and colleagues to measure the acculturation status for Asian or Korean American older adults who immigrated to the United States (Jang, Kim, Chiriboga, \& Kallimanis, 2007). Although this scale was originally developed for older adults, it includes major aspects of acculturation that are common, irrespective of age. The American Orientation Subscale evaluates how much individuals adopt the beliefs and behaviors of American culture, and it contains 12 items on English proficiency, frequency of English use, consumption of audiovisual media (e.g., TV or videos), consumption of printed media (e.g., newspaper or magazine), consumption of American food at home, consumption of 
American food outside the home, ethnicity of friends, social gathering, sense of belonging, getting along with Americans, familiarity with American culture and custom, and celebration of American holidays (Jang et al., 2007). A 4-point Likert-type scale ranging from 0 (low acculturation) to 3 (high acculturation) was used, which produces total scores with a range from 0 to 36, with lower scores indicating less adherence or acculturation to the American culture. Previously reported internal consistency was Cronbach's alpha .77 (Jang et al., 2007). The internal consistency and test-retest reliability in the current study were .87 and .93 , respectively.

Perceived Stress. Perceived stress, a measure of psychological stress, was assessed with the 10-item Perceived Stress Scale (PSS). This scale measures the overall amount of stress in one's life, and the items are designed to assess how unpredictable, uncontrollable, and overloaded respondents find their lives (Cohen, Kamarck, \& Mermelstein, 1983). A 5-point Likert-type scale from 0 (never) to 4 (very often) was used, and total scores can range from 0 to 40 , with lower scores indicating lower perceived stress. The 10-item PSS has good internal consistency (Cronbach's $\alpha=.85$ ), testretest reliability ( $r=.85$; Cohen et al., 1983), and construct validity and predictive validity (Cohen, Tyrrell, \& Smith, 1993). In the current study, internal consistency was Cronbach's alpha .83 and test-retest reliability was .79.

Acculturative Stress. Acculturative stress, another measure of psychological stress, was assessed with the 24-item Revised Social, Attitudinal, Familial, and Environmental scale (R-SAFE). The R-SAFE scale measures acculturationrelated stress, perceived discrimination, and majority group stereotypes toward immigrant populations (Mena, Padilla, \& Maldonado, 1987; Padilla, Wagatsuma, \& Lindholm, 1985). A 4-point Likert-type scale ranging from 0 (not stressful) to 3 (extremely stressful) was used, and total scores range from 0 to 72 , with higher scores indicating greater stress. Studies have reported that the R-SAFE has good construct validity (Mena et al., 1987) and convergent and discriminant validity (Joiner \& Walker, 2002), and that the R-SAFE is also reliable for Asian Americans (Cronbach's alpha .89; M. J. Kim, Ahn, Chon, Bowen, \& Khan, 2005). In the current study, the internal consistency as measured with Cronbach's alpha and testretest reliability were .88 and .73 , respectively.

Anxiety. Anxiety, a measure of stress-related emotions, was assessed with the State Anxiety Subscale of the Spielberger State-Trait Anxiety Inventory. This 20-item subscale used a 4-point Likert-type scale, and the total scores ranged from 20 to 80, with higher scores indicating greater anxiety (Spielberger \& Vagg, 1984). The Spielberger State-Trait Anxiety Inventory has demonstrated construct and divergent validity and test-retest reliability (Spielberger \& Vagg, 1984). In the current study, the State Anxiety Subscale also demonstrated a good internal consistency (Cronbach's $\alpha=.93$ ).
Depression. Depression, another measure of stress-related emotions, was assessed with the Center for Epidemiological Studies Depression Scale (CES-D; Radloff, 1977). This 20 -item self-report instrument was developed to assess mood, somatic symptoms, and interpersonal relationships within the last week (Radloff, 1977). This 4-point scale measures the frequency of occurrence, ranging from 0 (rarely or none of the time) to 3 (most or all of the time). The total sum scores range from 0 to 60 , with higher scores indicating greater depressive symptoms. This scale has been reported to be valid (Myers \& Weissman, 1980) and reliable (Cronbach's as were from .89 to .91; Seto, Cornelius, Goldschmidt, Morimoto, \& Day, 2005). In the current study, the internal consistency of the CES-D was Cronbach's $\alpha=.93$, and the test-retest reliability was .68 .

Demographic Factors. Age, gender, time in United States, education, and household income were self-reported. Age and time in United States were recorded in years. Gender and household income were recorded as male/female and in US dollars, respectively. Education reflects level of education completed.

\section{Scale Translation Process}

Because Korean versions were not available for the $\mathrm{JH}$ Active Coping Scale, the 10-item PSS, and the 24-item version of the R-SAFE, these scales were translated to Korean based on the Brislin's translation model (Jones, Lee, Phillips, Zhang, \& Jaceldo, 2001). The first author of the current article, a native Korean speaker, translated each into Korean. Afterward, a native Korean speaker fluent in both Korean and English reviewed the translated versions and translated them back into English. An American professor, a native English speaker, determined whether the back-translated English version of the scales was equivalent to the original scales. Based on the feedback of the American professor, the first author modified the Korean version of the scales. These processes were repeated until the American professor considered the back-translated English versions equivalent to the original scales.

\section{Data Analysis}

Descriptive data were expressed as means and standard deviations $(S D)$ for continuous variables and as frequencies and percentages for categorical variables. Pearson correlation was used to examine how $\mathrm{JH}$ active coping related to all other variables. Plots involving all variables reported for bivariate correlations were generated and examined for linearity. Multiple regressions were used to examine the effect of JH active coping on acculturation and psychological health after controlling for demographic factors. To assess multicollinearity of independent variables, the variance inflation factor (VIF) was examined, where VIF $>10$ was 
Table I. Descriptive Statistics of Psychological Variables.

\begin{tabular}{lcccr}
\hline Variable & Mean \pm SD & Minimum & Maximum & Possible values \\
\hline John Henryism active coping & $42.04 \pm 5.68$ & 21.0 & 54.0 & $12-60$ \\
Acculturation & $17.78 \pm 5.83$ & 6.0 & 33.0 & $0-36$ \\
Perceived stress & $16.59 \pm 4.54$ & 6.0 & 29.0 & $0-40$ \\
Acculturative stress & $16.98 \pm 9.14$ & 1.0 & 40.0 & $0-72$ \\
Anxiety & $36.06 \pm 9.07$ & 20.0 & 68.0 & $20-80$ \\
Depressive symptoms & $11.29 \pm 7.76$ & 0 & 38.0 & $0-60$ \\
\hline
\end{tabular}

used to indicate multicollinearity. The internal consistencies of the scales were analyzed by checking Cronbach's alpha, and the test-retest reliability was examined by testing the Pearson correlation coefficient. Statistical significance was determined by $p<.05$. All analyses were conducted using SAS Version 9.2.

\section{Results}

\section{Demographic Results}

Participants included 41 men and 61 women. Their ages ranged from 21 to 60 years, and the mean age was 39.64 years $(S D=9.90)$. The average time in the United States was 10.07 years $(S D=7.40)$. More than half of the participants $(67.64 \%)$ had a university or higher education. Household income of less than $\$ 49,999$ per year was reported by $65.68 \%$ of the sample; $23.58 \%$ between $\$ 50,000$ and $\$ 99,999$; and $10.78 \%$ greater than $\$ 100,000$. The most frequently reported occupations were professional (34.31\%), home maker (16.67\%), laborer (14.70\%), and no job (9.80\%). Most of the sample $(71.57 \%)$ lived with a spouse, and 15 participants (14.71\%) were unmarried, living with another person.

\section{Descriptive and Correlation Results}

Distributions of psychological variables are displayed in Table 1 , and the bivariate correlations of $\mathrm{JH}$ active coping with all other variables are presented in Table 2. Among the demographic factors, only education was significantly correlated with $\mathrm{JH}$ active coping; individuals with higher education reported higher $\mathrm{JH}$ active coping scores $(r=.29, p=.003)$. With regard to psychological variables, higher JH active coping was significantly related to higher levels of acculturation $(r=.30, p=.002)$. Interestingly, $\mathrm{JH}$ active coping was significantly, and inversely, related to all the psychological variables (acculturation stress $[r=-.29, p=.003]$, perceived stress $[r=$ $-.38, p=<.0001]$, anxiety $[r=-.35, p=.0003]$, and depression $[r=-.28, p=.005])$, suggesting better psychological health among those demonstrating an active coping predisposition.

\section{Multiple Regression Results}

Table 3 represents the results of the multiple regressions assessing relationships of $\mathrm{JH}$ active coping with acculturation and psychological health after controlling for demographic factors. JH active coping was an independent predictor of acculturation level, along with age and time in United States. For acculturative stress, age and $\mathrm{JH}$ active coping were significant predictors. Older participants reported more acculturative stress $(\beta=0.30, p=.003)$ and those with high $\mathrm{JH}$ active coping scores reported less acculturative stress $(\beta=$ $-0.36, p=.029)$. JH active coping was the only significant predictor of lower perceived stress scores $(\beta=-0.29, p<$ $.001)$, lower anxiety $(\beta=-0.42, p=.011)$, and lower depressive symptoms $(\beta=-0.32, p=.027)$. All values of VIF were less than 2 , suggesting that multicollinearity was not an issue.

\section{Discussion}

This study aimed to assess the levels of $\mathrm{JH}$ active coping and its relationship with acculturation and psychological health among Korean immigrants to the United States. Findings indicated that the levels of JH active coping in Korean immigrants may be lower than the levels reported in other racial groups including African Americans, European Americans, and Hispanic Americans; nevertheless, JH active coping was a significant and independent predictor of higher acculturation and better psychological health as denoted by lower levels of acculturative stress, perceived stress, anxiety, and depressive symptoms. These associations persisted after controlling for age, gender, time in the United States, education, and household income.

\section{John Henryism Active Coping in Korean Immigrants}

Individuals who score high in $\mathrm{JH}$ active coping believe that they can manage daily stressors and accomplish goals through hard work and determination (James et al., 1983; James et al., 1987; James, Keenan, Strogatz, Browning, \& Garrett, 1992). The levels of JH active coping observed in this sample were compared with scores reported in other studies conducted with similarly aged persons in different racial groups (Table 4). Although it is not possible to conduct statistical analysis due to limited information from other studies, the mean $\mathrm{JH}$ active coping for participants in this study (42.56 for men and 41.69 for women) was lower than scores reported for other racial groups (Bohman et al., 2009; A. F. Fernander et al., 2005; Haritatos et al., 2007; James 


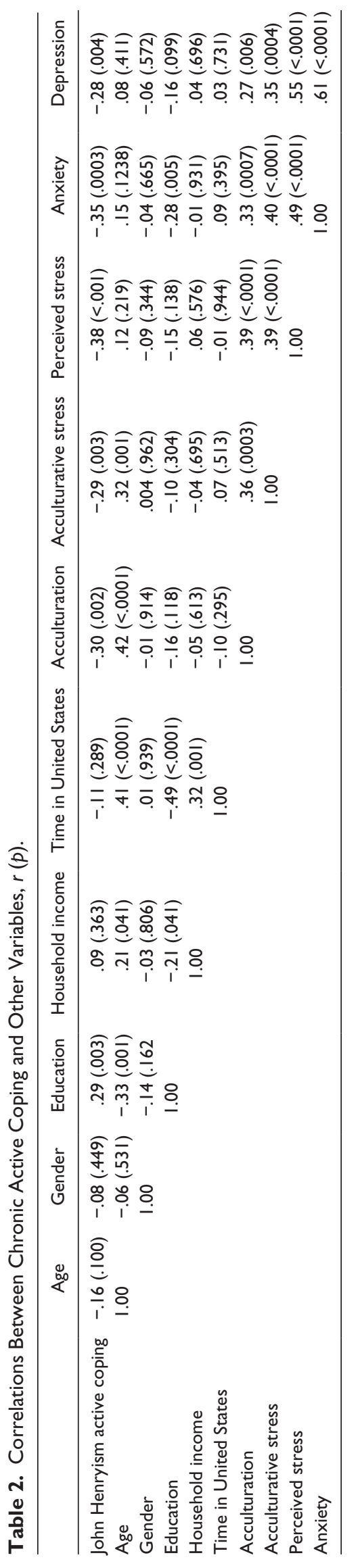




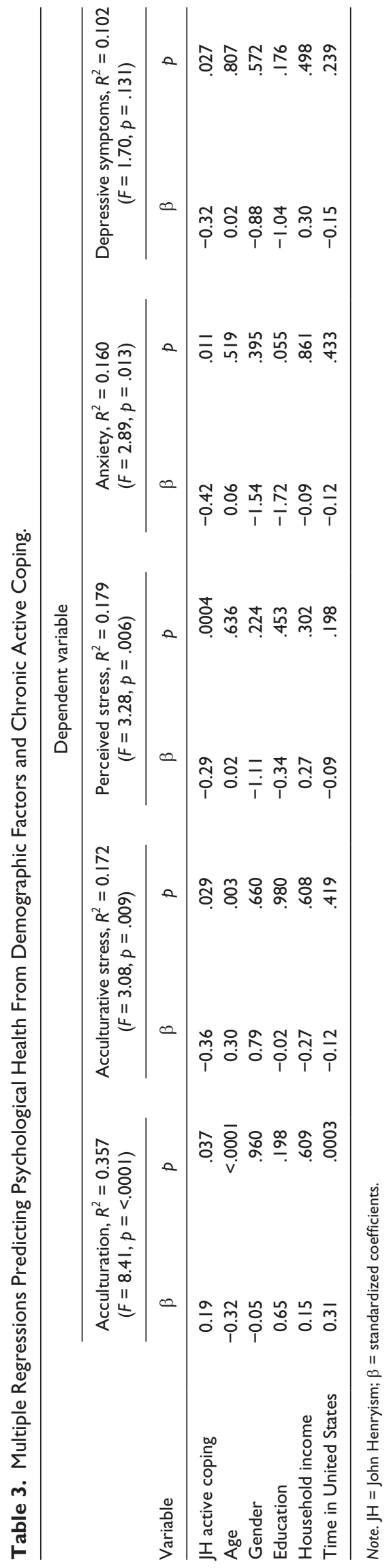




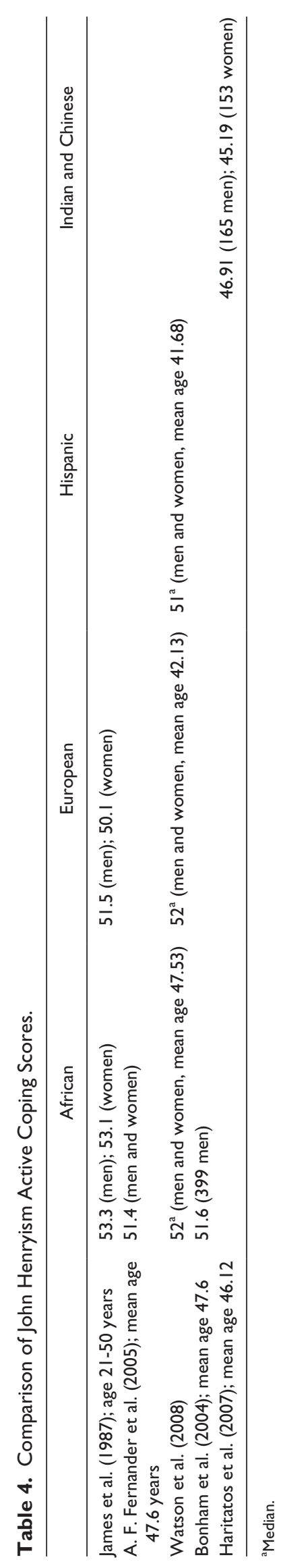


et al., 1987; Watson, Logan, \& Tomar, 2008). Among demographic factors, JH active coping was significantly, and positively, related only to education (Table 2). Given that the sample of Korean immigrants in this study was highly educated, perhaps a larger cross-section of Korean immigrants would have lower JH active coping scores.

Since individuals' stress appraisal and coping process are greatly influenced by their culture (Post \& Weddington, 1997), the relatively low JH active coping found in this Korean immigrant sample may be understood in the context of Korean culture. Although Western culture and values have had a great impact on the lives of modern-day Koreans, the philosophy and perspective of Confucianism are still found in the way Koreans perceive and cope with stressful events (Cha \& Kim, 2013; Park \& Chesla, 2007). Confucianism values social harmony, conformity, and respect for hierarchy of familial or social structure (Keum, 2003). These values may lead individuals to be more reluctant to assert their rights, to question those in authority, and to compromise or sacrifice their ways of handling daily stressors. It has been reported that Korean families are reluctant to seek public help due to fear that this could be perceived as a sign of a weakness or as dishonor to their family or group (Cho et al., 2009). Culture influenced by Confucianism is quite distinguishable from $\mathrm{JH}$ active coping, which emphasizes an enterprising spirit and optimism and discourages passivity, particularly in the face of adversity. Since most of the participants in this study are first-generation immigrants, they may be deeply influenced by Confucianism-oriented Korean values, which emphasize acceptance of, as opposed to battling with, life's adversities.

\section{John Henryism Active Coping and Acculturation}

Korean immigrants in the current study have lived in the United States an average of 10 years, and their acculturation level (17.78) was slightly lower than the middle score of the scale. Low acculturation level may denote less adherence to the American culture and more conflict between Korean and American cultures. Although bivariate correlation results showed that, of all demographic factors, acculturation was related only to age, further analysis showed that acculturation was significantly related to time in United States after controlling for the impact of age on acculturation $(r=.34$, $p<.0006)$. This supports the notion that over time, immigrants may become familiar with or adapt more to mainstream society and have less conflict between their host culture and native culture. Whereas it is presumed that participants with more socioeconomic resources may acculturate more easily, acculturation level was not related to the levels of education $(r=.16, p=.118)$ or income $(r=0.06, p=.526)$; nor did acculturation scores differ between men and women $(t=.11$, $p=.914)$. Moreover, multivariate analysis showed that $\mathrm{JH}$ active coping was an important determinant of acculturation levels after controlling for age and time in the United States.
Since this is a cross-sectional study, it is not clear which came first - higher JH active coping or higher acculturation; however, given that $\mathrm{JH}$ active coping was not correlated with time in the United States $(r=-0.11, p=.289)$, it is plausible that $\mathrm{JH}$ active coping may have spurred the acculturation process in this Korean immigrant group.

Acculturation status was negatively associated with all the indices of psychological distress, including perceived stress $(r=-0.40, p<.0001)$, acculturative stress $(r=-.36, p=$ $.0003)$, anxiety $(r=-.33, p=.0007)$, and depression $(r=-.27$, $p=.006)$. A causal relationship between acculturation status and psychological health requires further investigation.

\section{John Henryism Active Coping and Psychological Health}

The current study of Korean immigrants found that JH active coping is a significant and independent predictor of positive psychological health. This result is consistent with the previous study in which high JH predicted better self-rated health, in part, by reducing perceived stress among high SES Chinese and Indian immigrants (Haritatos et al., 2007). However, another study reported a significant negative association between $\mathrm{JH}$ and happiness in African American men after controlling for demographic factors (Angner, Hullett, \& Allison, 2011). It is not clear whether intersample differences in race, gender, and/or SES are the primary factors responsible for these inconsistent results, because SES is not always compatible across different studies. In addition, there is evidence that $\mathrm{JH}$ active coping is adaptive and health promoting in both African Americans men and women (Bonham et al., 2004; Bronder, Speight, Witherspoon, \& Thomas, 2014; Derogatis, Abeloff, \& Melisaratos, 1979; A. Fernander, Wilson, Staton, \& Leukefeld, 2005; Lehto \& Stein, 2013; Orr, James, Garry, \& Newton, 2006). Some of these studies examined how JH active coping was related to health behaviors, not stress and emotions, but they nevertheless help illuminate the sociopsychological context where high as opposed to low JH scores might be observed. This sample of Korean immigrants has high level of education but relatively low level of household income; therefore, it is not clear whether they belonged to either high or low SES. There is a need that the effect of JH active coping on psychological health be systematically scrutinized considering the confounding effect of demographic factors such as gender, race, and SES.

Our findings on $\mathrm{JH}$ active coping and psychological health are particularly meaningful due to the relatively high levels of psychological stress and depression reported in studies of Korean immigrants (Chun et al., 2007; M. T. Kim et al., 2005; Koh, 1998; Kuo, 1984; Shin, 1993). In accordance with previous studies, this sample of Korean immigrants seems to have high levels of perceived stress and depressive symptoms. As previously reported by our group (Logan, Barksdale, Carlson, Carlson, \& Rowsey, 2012), the mean of perceived stress in the current study (16.59) was 
higher than the reference values for Whites (12.8) and Blacks (14.7). Regarding depression, the mean score on the CES-D was 11.29 in this sample. However, one study that examined depressive symptoms with the CES-D scale in 117 breast cancer patients and 62 healthy people (mostly White Americans) prior to treatment and midway through treatment (Hann, Winter, \& Jacobsen, 1999) reported that mean scores on the CES-D were 10.9 and 12.8 for patients and 8.1 and 7.8 for healthy participants. Interestingly, this sample of Korean immigrants seems to have even more depressive symptoms than the cancer patients prior to undergoing treatment. This result is in agreement with a previous study of 499 Asian Americans, which observed that Korean Americans had higher levels of depression than Chinese, Filipinos, or Japanese Americans and reported that Korean Americans experience an "excessive amount of adjustment problems" (Kuo, 1984).

Our data demonstrate the extent of psychological stress and depression in Korean immigrants. Given this study finding on JH active coping as a significant predictor of psychological health, enhancing active coping with stressors could therefore be a useful intervention to promote healthy acculturation and greater psychological well-being in Korean immigrants. Active coping may include becoming aware of the problems, seeking advice or help, and taking actions to get rid of the problems. These interventions must be fully tested to determine the best ways to enhance $\mathrm{JH}$ active coping skills.

\section{Study Limitations and Strengths}

The current study has several limitations, the first of which is sampling bias. Study participants were recruited from three adjacent counties in North Carolina, which limits the generalizability of the findings. Second, many study participants were church goers; hence, habitual non-church goers might not be represented in the sample. Third, the age range of this study sample is wide and may hinder generalizations of the study results. Fourth, the cross-sectional design precludes drawing causal inferences regarding $\mathrm{JH}$ active coping, acculturation, and psychological health. Fifth, all psychological variables were self-reported and the magnitude of social desirability effects on the observed relationships is unknown.

Despite these limitations, this study has a number of strengths. First, several widely used psychosocial instruments of well-established clinical relevance were successfully translated from English to Korean and were shown to have acceptably high internal and test-retest reliability. Second, our research findings extend work on JH active coping with psychological stress to Korean immigrants, a group known to encounter cultural conflicts after arriving in the United States, and for whom sound theoretical models are needed to characterize their adaptation process. The current study opens a window on theoretical models that may explain the interaction of acculturation, psychological stress, and active coping in this group. Third, and most important, the study results indicating that the observed positive relationships of JH active coping with acculturation, on the one hand, and with psychological well-being on the other, are robust to statistical adjustments for demographic factors including age, gender, education, income, and time in the United States.

\section{Conclusion}

This study highlights new understanding of Korean immigrants' skills in adapting to a new culture. They appear to have lower JH active coping levels than other racial groups. Nevertheless, JH active coping are considered to enhance acculturation and psychological health in this Korean immigrant group. Efforts to identify those with low active coping early in their adjustment period and intervention designed to strengthen active coping could result in more successful acculturation and psychological health. Future studies are warranted to replicate the beneficial effect of active coping on acculturation and psychological health in a large sample of Korean immigrants and to develop preventive measures of adjustment problems in this population who are in need of such interventions.

\section{Declaration of Conflicting Interests}

The author(s) declared no potential conflicts of interest with respect to the research, authorship, and/or publication of this article.

\section{Funding}

The author(s) disclosed receipt of the following financial support for the research, authorship, and/or publication of this article: This study used the data from the study funded by Ruth L. Kirschstein National Research Service Award (F31NR011377) by the National Institute of Nursing Research (NINR)/National Institutes of Health (NIH).

\section{References}

Angner, E., Hullett, S., \& Allison, J. (2011). "I'll die with the hammer in my hand": John Henryism as a predictor of happiness. Journal of Economic Psychology, 32, 357-366. doi:10.1016/j. joep.2011.01.002

Bohman, H., Jonsson, U., Knorring, A. L., Knorring, L. V., Olsson, G., Paaren, A., . . . Naessen, T. (2009). Thicker carotid intima layer, thinner media layer and higher intima/ media ratio in women with recurrent depressive disorders: A pilot study using non-invasive high frequency ultrasound. World Journal of Biological Psychiatry, 11(1), 71-75. doi:10.1080/15622970902789122

Bonham, V. L., Sellers, S. L., \& Neighbors, H. W. (2004). John Henryism and self-reported physical health among high-socioeconomic status African American men. American Journal of Public Health, 94, 737-738.

Bronder, E. C., Speight, S. L., Witherspoon, K. M., \& Thomas, A. J. (2014). John Henryism, depression, and perceived social support in Black women. Journal of Black Psychology, 40, 115-137. doi:10.1177/0095798412474466 
Cha, C., \& Kim, E. (2013). Assessing the role of culture in Korean goose mothers' lives. Journal of Transcultural Nursing, 24, 86-93. doi:10.1177/1043659612452002

Cho, S. J., Lee, J. Y., Hong, J. P., Lee, H. B., Cho, M. J., \& Hahm, B. J. (2009). Mental health service use in a nationwide sample of Korean adults. Social Psychiatry and Psychiatric Epidemiology, 44, 943-951. doi:10.1007/s00127-009-0015-7

Chun, M., Knight, B. G., \& Youn, G. (2007). Differences in stress and coping models of emotional distress among Korean, Korean-American and White-American caregivers. Aging \& Mental Health, 11, 20-29. doi:10.1080/13607860600736232

Claassen, D., Ascoli, M., Berhe, T., \& Priebe, S. (2005). Research on mental disorders and their care in immigrant populations: A review of publications from Germany, Italy and the UK. European Psychiatry, 20, 540-549. doi:10.1016/j. eurpsy.2005.02.010

Cohen, S., Kamarck, T., \& Mermelstein, R. (1983). A global measure of perceived stress. Journal of Health and Social Behavior, 24, 385-396.

Cohen, S., Tyrrell, D. A., \& Smith, A. P. (1993). Negative life events, perceived stress, negative affect, and susceptibility to the common cold. Journal of Personality and Social Psychology, 64, 131-140.

Derogatis, L. R., Abeloff, M. D., \& Melisaratos, N. (1979). Psychological coping mechanisms and survival time in metastatic breast cancer. Journal of the American Medical Association, 242, 1504-1508.

Duijkers, T. J., Drijver, M., Kromhout, D., \& James, S. A. (1988). "John Henryism" and blood pressure in a Dutch population. Psychosomatic Medicine, 50, 353-359.

Fazel, M., Wheeler, J., \& Danesh, J. (2005). Prevalence of serious mental disorder in 7000 refugees resettled in western countries: A systematic review. Lancet, 365, 1309-1314. doi:10.1016/ S0140-6736(05)61027-6

Fernander, A., Wilson, J. F., Staton, M., \& Leukefeld, C. (2005). Exploring the type-of-crime hypothesis, religiosity, and spirituality in an adult male prison population. International Journal of Offender Therapy and Comparative Criminology, 49, 682-695. doi:10.1177/0306624X05274897

Fernander, A. F., Duran, R. E., Saab, P. G., \& Schneiderman, N. (2004). John Henry active coping, education, and blood pressure among urban blacks. Journal of the National Medical Association, 96, 246-255.

Fernander, A. F., Patten, C. A., Schroeder, D. R., Stevens, S. R., Eberman, K. M., \& Hurt, R. D. (2005). Exploring the association of John Henry active coping and education on smoking behavior and nicotine dependence among Blacks in the USA. Social Science \& Medicine, 60, 491-500. doi:10.1016/j.socscimed.2004.06.004

Folkman, S., \& Moskowitz, J. T. (2004). Coping: Pitfalls and promise. Annual Review of Psychology, 55, 745-774. doi:10.1146/ annurev.psych.55.090902.141456

Glozman, J. M., \& Markovna, Z. (2004). Communication disorders and personality. New York, NY: Springer Science.

Han, H. R., Kim, M., Lee, H. B., Pistulka, G., \& Kim, K. B. (2007). Correlates of depression in the Korean American elderly: Focusing on personal resources of social support. Journal of Cross-Cultural Gerontology, 22, 115-127. doi:10.1007/ s10823-006-9022-2
Hann, D., Winter, K., \& Jacobsen, P. (1999). Measurement of depressive symptoms in cancer patients: Evaluation of the Center for Epidemiological Studies Depression Scale (CESD). Journal of Psychosomatic Research, 46, 437-443.

Haritatos, J., Mahalingam, R., \& James, S. A. (2007). John Henryism, self-reported physical health indicators, and the mediating role of perceived stress among high socio-economic status Asian immigrants. Social Science \& Medicine, 64, 1192-1203. doi:10.1016/j.socscimed.2006.10.037

James, S. A. (1994). John Henryism and the health of AfricanAmericans. Culture, Medicine, and Psychiatry, 18, 163-182.

James, S. A., Hartnett, S. A., \& Kalsbeek, W. D. (1983). John Henryism and blood pressure differences among black men. Journal of Behavioral Medicine, 6, 259-278.

James, S. A., Keenan, N. L., Strogatz, D. S., Browning, S. R., \& Garrett, J. M. (1992). Socioeconomic status, John Henryism, and blood pressure in black adults. The Pitt County Study. American Journal of Epidemiology, 135, 59-67.

James, S. A., Strogatz, D. S., Wing, S. B., \& Ramsey, D. L. (1987). Socioeconomic status, John Henryism, and hypertension in blacks and whites. American Journal of Epidemiology, 126, 664-673.

Jang, Y., Kim, G., \& Chiriboga, D. (2005). Acculturation and manifestation of depressive symptoms among KoreanAmerican older adults. Aging \& Mental Health, 9, 500-507. doi:10.1080/13607860500193021

Jang, Y., Kim, G., Chiriboga, D., \& Kallimanis, B. (2007). A bidimensional model of acculturation for Korean American older adults. Journal of Aging Studies, 21, 267-275. doi:10.1016/j. jaging.2006.10.004

Joiner, T. E., Jr., \& Walker, R. L. (2002). Construct validity of a measure of acculturative stress in African Americans. Psychological Assessment, 14, 462-466.

Jones, P. S., Lee, J. W., Phillips, L., Zhang, X., \& Jaceldo, K. (2001). An adaptation of Brislin's translation model for crosscultural research. Nursing Research, 50, 300-304.

Keum, J. (2003). Hyundae Hanguk Yookyo-way Jyontong [Adaptation of Confucian organizations to contemporary Korean Society]. Seoul, South Korea: Seoul National University Press.

Kim, M. J., Ahn, Y. H., Chon, C., Bowen, P., \& Khan, S. (2005). Health disparities in lifestyle choices among hypertensive Korean Americans, non-Hispanic Whites, and Blacks. Biological Research for Nursing, 7, 67-74. doi:10.1177/1099800405276820

Kim, M. T., Han, H. R., Shin, H. S., Kim, K. B., \& Lee, H. B. (2005). Factors associated with depression experience of immigrant populations: A study of Korean immigrants. Archives of Psychiatric Nursing, 19, 217-225.

Koh, K. B. (1998). Perceived stress, psychopathology, and family support in Korean immigrants and nonimmigrants. Yonsei Medical Journal, 39, 214-221.

Kramer, N. M., Johnson, N. L., \& Johnson, D. M. (2015). Is John Henryism a resilience factor in women experiencing intimate partner violence? Anxiety, Stress, and Coping, 28, 601-616. doi:10.1080/10615806.2014.1000879

Kuo, W.H. (1984). Prevalence of depression among Asian-Americans. Journal of Nervous and Mental Disease, 172, 449-457.

Lazarus, R. S., \& Folkman, S. (1984). Stress, appraisal, and coping. New York, NY: Springer. 
Lehto, R. H., \& Stein, K. F. (2013). The impact of John Henryism on self-reported health behaviors in African American men. Journal of Transcultural Nursing, 24, 291-296. doi:10.1177/1043659613481673

Lin, K. M., Lau, J. K., Yamamoto, J., Zheng, Y. P., Kim, H. S., Cho, K. H., \& Nakasaki, G. (1992). Hwa-byung. A community study of Korean Americans. Journal of Nervous and Mental Disease, 180, 386-391.

Logan, J. G., Barksdale, D. J., Carlson, J., Carlson, B. W., \& Rowsey, P. J. (2012). Psychological stress and arterial stiffness in Korean Americans. Journal of Psychosomatic Research, 73, 53-58. doi:10.1016/j.jpsychores.2012.04.008

Mena, F. J., Padilla, A. M., \& Maldonado, M. (1987). Acculturative stress and specific coping strategies among immigrant and later generation college students. Hispanic Journal of Behavioral Sciences, 9, 207-225.

Merritt, M. M., Bennett, G. G., Williams, R. B., Sollers, J. J., 3rd, \& Thayer, J. F. (2004). Low educational attainment, John Henryism, and cardiovascular reactivity to and recovery from personally relevant stress. Psychosomatic Medicine, 66, 49-55.

Myers, J. K., \& Weissman, M. M. (1980). Use of a self-report symptom scale to detect depression in a community sample. American Journal of Psychiatry, 137, 1081-1084.

Orr, S. T., James, S. A., Garry, J., \& Newton, E. (2006). Exercise participation before and during pregnancy among low-income, urban, Black women: The Baltimore Preterm Birth Study. Ethnicity \& Disease, 16, 909-913.

Padilla, A. M., Wagatsuma, Y., \& Lindholm, K. J. (1985). Acculturation and personality as predictors of stress in Japanese and JapaneseAmericans. Journal of Social Psychology, 125, 295-305.
Park, M., \& Chesla, C. (2007). Revisiting Confucianism as a conceptual framework for Asian family study. Journal of Family Nursing, 13, 293-311. doi:10.1177/1074840707304400

Post, D. M., \& Weddington, W. (1997). The impact of culture on physician stress and coping. Journal of the National Medical Association, 89, 585-590.

Radloff, L. S. (1977). The CES-D Scale: A self-report depression scale for research in the general population. Applied Psychological Measurement, 1, 385-401.

Seto, M., Cornelius, M. D., Goldschmidt, L., Morimoto, K., \& Day, N. L. (2005). Long-term effects of chronic depressive symptoms among low-income childrearing mothers. Maternal and Child Health Journal, 9, 263-271. doi:10.1007/s10995-005-0002-x

Shin, K. R. (1993). Factors predicting depression among KoreanAmerican women in New York. International Journal of Nursing Studies, 30, 415-423.

Spielberger, C. D., \& Vagg, P. R. (1984). Psychometric properties of the STAI: A reply to Ramanaiah, Franzen, and Schill. Journal of Personality Assessment, 48, 95-97. doi:10.1207/ s15327752jpa4801_16

Watson, J. M., Logan, H. L., \& Tomar, S. L. (2008). The influence of active coping and perceived stress on health disparities in a multi-ethnic low income sample. BMC Public Health, 8, 41. doi:10.1186/1471-2458-8-41

Wigg, S., Wright, E., Breach, P., \& Wilson, J. D. (1996). Is it diabetes mellitus or Munchausen's syndrome? Australian and New Zealand Journal of Medicine, 26(6), 841.

Wiist, W. H., \& Flack, J. M. (1992). A test of the John Henryism hypothesis: Cholesterol and blood pressure. Journal of Behavioral Medicine, 15(1), 15-29. 